\title{
A possible connection between gamma-ray bursts and single black holes
}

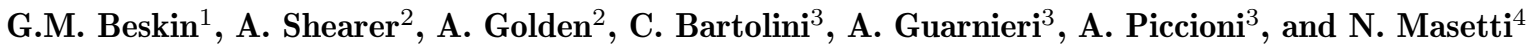 \\ 1 Special Astrophysical Observatory, Russia \\ 2 University of Galway, Ireland \\ 3 Dipartimento di Astronomia, Università di Bologna, via Zamboni 33, I-40126 Bologna, Italy \\ 4 Istituto Te.S.R.E., CNR, Bologna, Italy
}

Received December 18, 1998; accepted July 1, 1999

\begin{abstract}
We consider Single Black Holes (SBHs) of $10^{3} M_{\odot}$ as possible parent objects for cosmological GRBs. The latter are the result of the energy release of SBHs magnetosphere inhomogeneities. The percolation nature of this release yields a unique power law for the GRBs energy distribution that is $E^{-\beta}$ (with $\beta=1.7-1.8$ ), i.e. the same observed in solar flares, flare stars and X-ray sources. The number of GRBs per year per galaxy is about $10^{-2}-10^{-1}$ with total energy $10^{47}-10^{50}$ ergs for beaming factor of about $10^{-4}-10^{-5}$.
\end{abstract}

Key words: black hole physics, gamma-rays: bursts

\section{Introduction}

There is much evidence that most GRBs have cosmological origin (see Piran 1998 for a recent review). Observations give total isotropic energy fluences of these events in the range $10^{51}-10^{54}$ erg during several seconds. From BATSE observations, the GRBs rate is one burst per million years per galaxy. The energy release and rate can be reduced, of course, by several orders of magnitude if the GRBs are beamed with a factor of about of $10^{-4}-10^{-5}$. The central point in the GRBs scenario is the origin of the "inner engine" that we do not detect directly. A significant difficulty with many proposed parent bodies of GRBs is that only a small amount of baryons $\left(<10^{-5} M_{\odot}\right)$ can be involved if a required relativistic expansion is to be produced (Shemi \& Piran 1990). Scenarios using coalescence of two neutron stars (Narayan et al. 1992) or special types of supernovae (Paczyński 1998) involve potentially large amounts of "contaminating" baryons. A baryon-poor mechanism is based on the possibility to convert rotational energy of neutron stars due to strong magnetic field $\left(10^{15} \mathrm{G}\right)$ to a Poynting flux and then, to kinetic energy of particles (see e.g. Usov 1992). We proposed as similar sources of magnetic energy, the inhomogeneities in the magnetospheres of Single Black Holes $(\mathrm{SBH})$ of $\left(10-10^{3}\right) M_{\odot}$ (Beskin et al.

Send offprint requests to: bartolini@astbo3.bo.astro.it
1998). These objects can be parent bodies of both galactic and cosmological GRBs.

In this paper we discuss in detail a possible connection between cosmological GRBs and SBHs.

\section{Assumptions for the GRB mechanism}

During accretion of interstellar plasma onto a SBH, magnetic fields strengthen up to $10^{11}-10^{12} \mathrm{G}$ and probably form a regular magnetosphere (Bisnovatyj-Kogan \& Ruzmaikin 1976; Kardashev 1995; Chakrabarti 1996; Beskin 1997).

In the magnetosphere there are local inhomogeneities similar to those of Sun and flare stars (Byrne 1992; Priest 1994) but with magnetic fields up to $10^{14} \mathrm{G}$.

The energy of these inhomogeneities as the result of spontaneous development of plasma instabilities turns into energy of fast particles and into anomalous heating. The GRB is therefore the burst of photons that have been generated as the result of such a process. We think this is an analog of solar flares and flashes of UV Ceti type stars and of the activity in $\mathrm{X}$-ray binaries with compact components (for some evidence of this see Katsova \& Livshits 1991; Rotschild et al. 1974; Meekins 1984; Bartolini et al. 1994).

The distribution of these flares (as well as GRBs) as a function of energy has a universal character, i.e. $\frac{\mathrm{d} N(E)}{\mathrm{d} E} \propto E^{-\beta}$, where $\beta=1.7-1.8$. Such a universality is caused by the percolation nature of the stability break in active regions (Feder 1988; Wentzel \& Seiden 1992; Pustil'nik 1997). Electrons (or positrons) and protons are accelerated in processes of dissipation of magnetic energy inhomogeneities up to $\gamma \sim 10^{3}-10^{6}$. Particle streams are beamed as result of one- and two-dimensions structure of magnetic field reconnection regions. They can change the direction of the motion very rapidly. The radiation is generated by these particles in magnetic fields near SBHs and in the interstellar medium (ISM). The emission is beamed as result of the stream sharpness and of the relativistic motion. 


\section{Energy and number of GRBs}

The SBHs under consideration are remnants of Population I-II and III stars located in the disc or in the halo of galaxies. In the first case their masses are greater than $10 M_{\odot}$, while in the second are greater than $10^{3} M_{\odot}$. In this hypothesis, SBHs are made of baryonic dark matter and have integral mass about $(0.1-1) M_{\text {gal }} \sim\left(10^{9}-10^{10}\right) M_{\odot}$; this means that the number of SBHs is $10^{6}-10^{8}$ per galaxy. These estimates come from dynamical, photometric and chemical data (Bahcall 1984; Carr 1994). Of course the density of SBHs has to be higher in galaxies with starforming regions.

We show here that the maximum energy of cosmological GRBs can be accumulated in magnetospheric inhomogeneities near massive $\left(M \gtrsim 10^{3} M_{\odot}\right)$ SBHs. Observing isotropic energy of GRBs is $10^{51}-10^{54} \mathrm{ergs}$ (Piran 1998). There are many reasons to think that their radiation is beamed with a factor $\sigma=\theta^{2} / 4 \pi \sim 10^{-4}-10^{-5}$. $\theta$ is either angular size of particles stream or beaming angle of these particles radiation $(1 / \gamma)$ (Piran 1998). After taking into account this effect, the energy of GRBs becomes $10^{47}-10^{50}$ ergs in a rest frame. It was shown the $\mathrm{SBH}$ accretes the plasma from ISM with a rate (for recent review see Chakrabarti 1996) $\dot{M}=1.410^{13} n M_{3}^{2} V_{300}^{-3} \mathrm{~g} \mathrm{~s}^{-1}$, where $n$ is number density of ISM in units of $\mathrm{cm}^{-3}, M_{3}$ is the SBH mass in units of $10^{3} M_{\odot}, V_{300}$ is velocity of SBH in units of $300 \mathrm{~km} \mathrm{~s}^{-1}$. The magnetic field frozen in the plasma during accretion is reinforced and conserved near the horizon (e.g. Bisnovatyj-Kogan \& Ruzmaikin 1976; Macdonald et al. 1986; Kardashev 1995; Beskin 1997). There is not a self-consistent model of this process and we will estimate possible parameters for the SBH magnetosphere in a phenomenological frame only.

If the part of the full mass-energy of the accreted plasma $\delta \dot{M} c^{2}$ is stored in form of magnetosphere energy during the time $t$, then $\left(H_{\mathrm{g}} / 8 \pi\right)(4 / 3) \pi r_{\mathrm{g}}{ }^{3} \sim \delta \dot{M} c^{2} t$, where $H_{\mathrm{g}}$ value of global magnetic field, $r_{\mathrm{g}}=2 G M / c^{2}$, and $H_{\mathrm{g}} \sim 310^{12} \delta_{-1}^{1 / 2} M_{3}{ }^{-1 / 2} V_{300}^{-3 / 2} n^{1 / 2} t_{9}^{1 / 2} \mathrm{G}$, where $\delta_{-1}$ is expressed in units of 0.1 , and $t$ in units of $10^{9}$ years.

For a magnetic field $H_{1}$ in inhomogeneities of size $r$ for constant magnetic flux it is easy to obtain that: $H_{\mathrm{l}} \sim H_{\mathrm{g}}\left(r_{\mathrm{g}} / r\right)^{2} \sim 310^{14} \alpha_{0.1}^{-2} \delta_{-1}^{1 / 2} M_{3}^{-1 / 2} V_{300}^{-3 / 2} n^{1 / 2} t_{9}^{1 / 2} \mathrm{G}$, where $\alpha=r_{\mathrm{g}} / r$ is in units of 0.1 .

For the magnetic energy concentrated in the inhomogeneities we obtain $E_{1} \sim\left(H_{1}^{2} / 8 \pi\right) \cdot(4 / 3) \pi r^{3} \sim$ $410^{50} \alpha_{0.1}^{-1} M_{3}^{3} \delta_{-1} n V_{300}^{-3} t_{9}$ ergs. This means that this amount of energy, accumulated during $10^{9}$ years, can be released as observable GRBs. We estimate that the mass of matter trapped in the inhomogeneity region during the time $t$ is

$m=\dot{M}\left(r / r_{\mathrm{g}}\right)^{3} t \sim 4.310^{26} n M_{3}^{2} V_{300}^{-3} \alpha_{0.1}^{3} t_{9} \sim 2$ $10^{-7} M_{\odot} n M_{3}^{2} V_{300}^{-3} \alpha_{0.1}^{3} t_{9}$.

Only $210^{-7} M_{\odot}$ is accumulated during $10^{9}$ years. This means that the baryon contamination is absent. In the results of instabilities development in inhomogeinity its magnetic energy converts to kinetic energy of electrons and protons. If $\beta$ is the efficiency of this conversion, then $\beta n_{\mathrm{e}} \gamma_{\mathrm{e}} m_{\mathrm{e}} c^{2} \sim \beta n_{\mathrm{p}} \gamma_{\mathrm{p}} m_{\mathrm{p}} c^{2} \sim\left(H_{\mathrm{l}}^{2}\right) / 8 \pi$, and we obtain $\gamma_{\mathrm{e}} \sim 10^{6} \beta, \gamma_{\mathrm{p}} \sim 10^{3} \beta$. The stream of particles is extremely relativistic as it is necessary to explain the GRBs observational properties.

We postulate a universal form for flares number distribution with energy if they are connected with the magnetosphere in inhomogeneities. This means that the parameter $k$ of the distribution $\mathrm{d} N(E) / \mathrm{d} E=k E^{-\beta}(\beta \sim 1.7)$ is the same for solar and UV Ceti flares stars and GRBs, they have different energy ranges: $10^{27}-10^{32}$ ergs for Sun and UV Ceti stars, $10^{47}-10^{50}$ ergs for GRBs. Taking $k \sim 610^{24} \mathrm{yr}^{-1}$ from observations of UV Ceti stars (Beskin et al. 1988) we obtain for a minimal GRBs energy of $10^{47}$ ergs (in the rest frame) about $10^{-8}$ flares per SBH per year. Using estimated number of SBH per galaxy, $10^{6}-10^{8}$, we have $10^{-2}-10^{0}$ GRBs per year per galaxy. Piran (1998) gives for this number $10^{-2}$ (for beaming factor $\left.\sigma \sim 10^{-4}\right)$. Taking into account all of our assumptions, this coincidence is very promising!

Acknowledgements. This investigation was supported by the Russian Fund of Fundamental Research, by the Educational Scientific Centre "Cosmion", by the italian Ministry of Foreign Affairs and by the University of Bologna (Funds for selected research topics).

\section{References}

Bahcall J., 1984, ApJ 219, 1008

Bartolini C., et al., 1994, ApJS 92, 455

Beskin V.S., 1997, Uspechi Fiz. Nauk 167, 689, Gershberg R.E. (eds.). Flares and Flashes, Springer, Berlin, p. 330

Beskin G.M., et al., 1998, in: Meegan C.A., Preece R.D., Koshut T.M. (eds.) 4th Huntsville GRB Symposium, AIP Conf. Proc. 428, 815

Beskin G.M., et al., 1988, Bull. KrAO 79, 71

Bisnovatyj-Kogan, Ruzmaikin, 1976, Ap. Space Sci. 42, 401

Byrne P.B., 1992, in: Linsky J.L., Serio S. (eds.) Physics of Solar \& Stellar Coronae. Kluwer, Dordrecht, p. 489

Carr B., 1994, ARA\&A 32, 531

Chakrabarti S.K., 1996, Phys. Rep. 266, 229

Feder J., 1988, Fractals. Plenum Press, New York

Kardashev N.S., 1995, MNRAS 276, 515

Katsova M.M., Livshits M.A., 1991, SvA 68, 131

Macdonald D.A., et al., 1986, in: Thorne K. et al. (eds.). Black Holes: The Membrane Paradigm, Yale Univ. Press

Meekins J.F., 1992, ApJ 278, 288

Narayan R., Paczynski B., Piran T., 1992, ApJ 395, L83

Paczynski B., 1998, ApJ 494, L45

Piran T., 1998, Phys. Rep. (in press) (astroph/9810256)

Priest E.R., 1994, in: Greiner J., Dürbeck H.W., Gershberg R.E. (eds.). Flares and Flashes, Springer, Berlin, p. 3

Pustil'nik L.A., 1997, Ap. Space Sci. 252, 325

Rothschild R.E., et al., 1974, ApJ 189, L13

Shemi A., Piran T., 1990, ApJ 365, L55

Usov V.V., 1992, Nat 357, 472

Wentzel D.G., Seiden P.E., 1992, ApJ 390, 280 